Technological University Dublin

DÜBLIN

ARROW@TU Dublin

2010-01-01

\title{
A Study of the Effect of the Position of an Edge Filter Within a Ratiometric Wavelength Measurement System
}

\author{
Qiang wu \\ Technological University Dublin, qiang.wu@tudublin.ie \\ Pengfei Wang \\ Technological University Dublin, pengfei.wang@tudublin.ie \\ Yuliya Semenova \\ Technological University Dublin, yuliya.semenova@tudublin.ie
}

See next page for additional authors

Follow this and additional works at: https://arrow.tudublin.ie/engscheceart

Part of the Electrical and Computer Engineering Commons

\section{Recommended Citation}

Wu, Q. et al. (2010) A Study of the Effect of the Position of an Edge Filter Within a Ratiometric Wavelength System. Measurement Science and Technology, Vol. 21, no. 9. doi:10.1088/0957-0233/21/9/094013

This Article is brought to you for free and open access by the School of Electrical and Electronic Engineering at ARROW@TU Dublin. It has been accepted for inclusion in Articles by an authorized administrator of ARROW@TU Dublin. For more information, please contact arrow.admin@tudublin.ie, aisling.coyne@tudublin.ie, gerard.connolly@tudublin.ie.

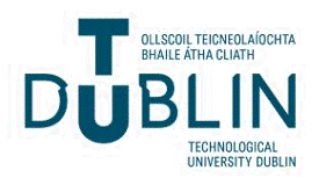


Authors

Qiang wu, Pengfei Wang, Yuliya Semenova, and Gerald Farrell

This article is available at ARROW@TU Dublin: https://arrow.tudublin.ie/engscheceart/88 


\title{
A study of the effect of the position of an edge filter within a ratiometric wavelength measurement system
}

\author{
Qiang Wu, Pengfei Wang, Yuliya Semenova and Gerald Farrell \\ Photonics Research Center, School of Electronic and Communications Engineering, Dublin Institute of \\ Technology, Kevin Street, Dublin 8, Ireland
}

Received 2 December 2009, in final form 23 March 2010

Published DD MMM 2010

Online at stacks.iop.org/MST/21/000000

\begin{abstract}
The effect of the position of an edge filter within a ratiometric wavelength measurement system was investigated based on three cases: (1) the reflected fibre Bragg grating (FBG) signal passes through both the reference arm and the edge filter arm, (2) the reflected FBG signal is connected directly to the edge filter arm and does not pass through the reference arm, (3) the edge filter sits in line with the FBG and thus the source power is filtered prior to reaching the FBG. Both numerical simulations and experimental results show that cases 1 and 2 have similar system performance whilst case 3 is the best arrangement which offers the highest wavelength resolution.
\end{abstract}

Keywords: ratiometric system, FBG, edge filter

Q1 (Some figures in this article are in colour only in the electronic version)

\section{Introduction}

The extraction of wavelength information in optical sensing systems is a very common function $[1,2]$. One particular example is fibre Bragg grating (FBG)-based optical sensing systems which have attracted wide interest because of their unique advantages such as immunity from electrical and magnetic interference, and chemical resistance [3]. It is widely used in bridge [4], river surveillance monitoring, civil structural monitoring and aerospace health monitoring [5], etc. In all these applications, monitoring of wavelength shifts is a critical function. There are various techniques for extracting wavelength information such as a ratiometric approach [6-8], scanning Fabry-Perot filters [9] and unbalanced MachZehnder interferometers [10]. Among these technologies, ratiometric wavelength measurement is a simple, high-speed and cost-effective scheme compared to other schemes. A detailed analysis of the effect of the optical source, signalto-noise ratio (SNR) and the transmission response of the edge filter has been provided in previous investigations [8]. However, to the best of our knowledge, there has been no report on an investigation of how to achieve a maximum resolution based on the arrangement of the existing components in a ratiometric system. How to use the existing components to achieve the best performance is very important in an optical sensing system. This paper describes an investigation of the effect of the position of the edge filter in a ratiometric wavelength measurement system. In section 2, three possible arrangements for the position of the edge filter within a ratiometric interrogation system are provided and numerical simulations were carried out. In section 3, experimental verification is reported using a FBG temperature sensor.

\section{System description}

There are three usable positions for the edge filter in a ratometric system. A schematic diagram of the three cases is shown in figures $1(a)-(c)$ : (1) the reflected FBG signal passes through both the reference arm and the edge filter arm, (2) the reflected FBG signal is connected directly to the edge filter arm and does not pass through the reference arm, (3) in this case the edge filter is moved so that it sits in line with the FBG and thus the source power is filtered prior to reaching the FBG.

All the three cases utilize a reference arm to compensate for the power fluctuations in the input optical source. 


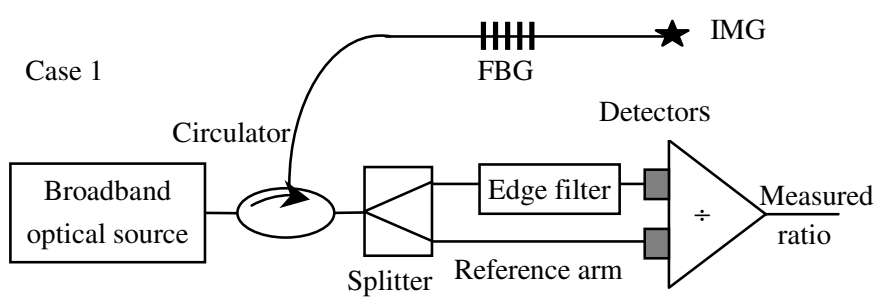

(a)

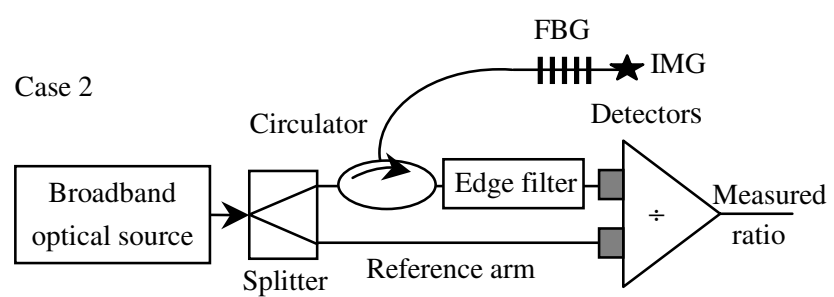

(b)

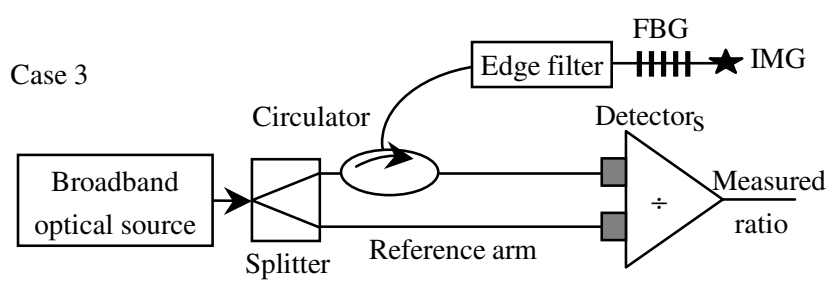

(c)

Figure 1. Schematic diagram of the ratiometric interrogation system with $(a)$ case $1,(b)$ case 2 and $(c)$ case 3 .

In case 1 as shown in figure $1(a)$, the reflected FBG signal is divided into two paths by a splitter: one goes directly to the photodiode acting as a reference signal, the other goes through an edge filter and detected by a photodiode. This is the common case in a ratiometric system [11]. A change in temperature or strain will alter the FBG reflected wavelength, and the power detected by the photodiode at the signal arm will change due to the response of the edge filter. The changed power ratio will allow extraction of the wavelength shift of the FBG. Case 2 as shown in figure $1(b)$ is similar to case 1 except for the use of the optical source power instead of the reflected FBG signal as a reference. In case 3, the edge filter was arranged between the circulator and FBG which means that the optical signal goes twice through the edge filter-this should increase the effective slope of the edge filter and hence improve the resolution of the ratiometric system.

The ratio of the ratiometric system in cases $1-3$ can be expressed as (1)-(3), respectively,

$$
\begin{gathered}
R\left(\lambda_{0}\right)=10 \log _{10}\left[\frac{\int I(\lambda) G_{\lambda_{0}}(\lambda) T(\lambda) \mathrm{d} \lambda+N_{e}}{\int I(\lambda) G_{\lambda_{0}}(\lambda) \mathrm{d} \lambda+N_{r}}\right] \\
R\left(\lambda_{0}\right)=10 \log _{10}\left[\frac{\int I(\lambda) G_{\lambda_{0}}(\lambda) T(\lambda) \mathrm{d} \lambda+N_{e}}{\int I(\lambda) \mathrm{d} \lambda+N_{r}}\right] \\
R\left(\lambda_{0}\right)=10 \log _{10}\left[\frac{\int I(\lambda) T(\lambda) G_{\lambda_{0}}(\lambda) T(\lambda) \mathrm{d} \lambda+N_{e}}{\int I(\lambda) \mathrm{d} \lambda+N_{r}}\right]
\end{gathered}
$$

where $I(\lambda)$ is the optical power spectral density of the broadband optical source, $T(\lambda)$ is the transmission response of an edge filter, $G_{\lambda_{0}}(\lambda)$ and $\lambda_{0}$ are the transmission response and the centre wavelength of the FBG, respectively, $\mathrm{Ne}$ and $\mathrm{Nr}$ are the background noise of the photodiodes at the arm of the edge filter and the reference signal, respectively.

Assume that the transmission response of an edge filter $T(\lambda)$ in a wavelength range from $\lambda_{1}$ to $\lambda_{2}$ is a triangular spectrum which can be expressed as

$$
\begin{aligned}
& 10 \log _{10}[T(\lambda)]=10 \log _{10}\left[T\left(\lambda_{1}\right)\right] \\
& +m\left|\lambda-\lambda_{d}\right| \quad \lambda_{1} \leqslant \lambda_{d} \leqslant \lambda_{2}
\end{aligned}
$$

where $m$ and $\lambda_{d}$ are the slope and peak wavelength of the edge filter, respectively.

The output spectral response of a FBG can be approximated by a Gaussian function with a $3 \mathrm{~dB}$ spectral width $\Delta \lambda_{0}$ and centre wavelength $\lambda_{0}$ [12]:

$10 \log _{10}\left[G_{\lambda_{0}}(\lambda)\right]=$

$$
\begin{cases}10 \log _{10} \exp \left[-4 \ln 2\left(\frac{\lambda-\lambda_{0}}{\Delta \lambda_{0}}\right)^{2}\right] & \left|\lambda-\lambda_{0}\right| \leqslant \Omega \lambda_{1} \leqslant \lambda \leqslant \lambda_{2} \\ -S & \left|\lambda-\lambda_{0}\right|>\Omega \lambda_{1} \leqslant \lambda \leqslant \lambda_{2}\end{cases}
$$

where $S$ is the SNR of the optical signal and $\Omega$ is determined by the optical source with a given noise level which can be expressed as

$$
10 \log _{10}\left\{\exp \left[-4 \ln 2\left(\frac{\Omega}{\Delta \lambda_{0}}\right)^{2}\right]\right\}=-S .
$$

\section{Numerical simulations}

\subsection{Influence of the spectral response of an optical source}

Since the output of an optical source at a wavelength range from 1500 to $1600 \mathrm{~nm}$ is not flat which could either be a constant or having a linear slope $m_{s}$. Investigations were firstly carried out on the influence of the spectral response of an optical source for all the three cases above and the simulation results are shown in figure 2. The parameters used in our simulations were $-90 \mathrm{dBm}$ as the equivalent background noise of both photodiodes and a $1 \mathrm{~dB}$ insertion loss for the edge filter, $\lambda_{1}=1540 \mathrm{~nm}, \lambda_{2}=1560 \mathrm{~nm}, \lambda_{d}=1550 \mathrm{~nm}, \Delta \lambda_{0}=1 \mathrm{~nm}$, $S=20 \mathrm{~dB}$ and $m=10 \mathrm{~dB} \mathrm{~nm}^{-1}$.

Figures $2(a)-(c)$ show that case 3 has the largest slope (hence the best resolution) no matter whether the slope of an optical source is a constant or a positive or a negative value. Figures $2(b)$ and $(c)$ indicate that when the slope response of the optical source has the same direction with that of the edge filter, the slope of the ratiometric system in case 2 is larger than that in case 1 . The maximum slopes in case 3 in figures $2(a)-$ (c) are 15.9, 16.58 and $-16.58 \mathrm{~dB} \mathrm{~nm}^{-1}$ respectively, which 

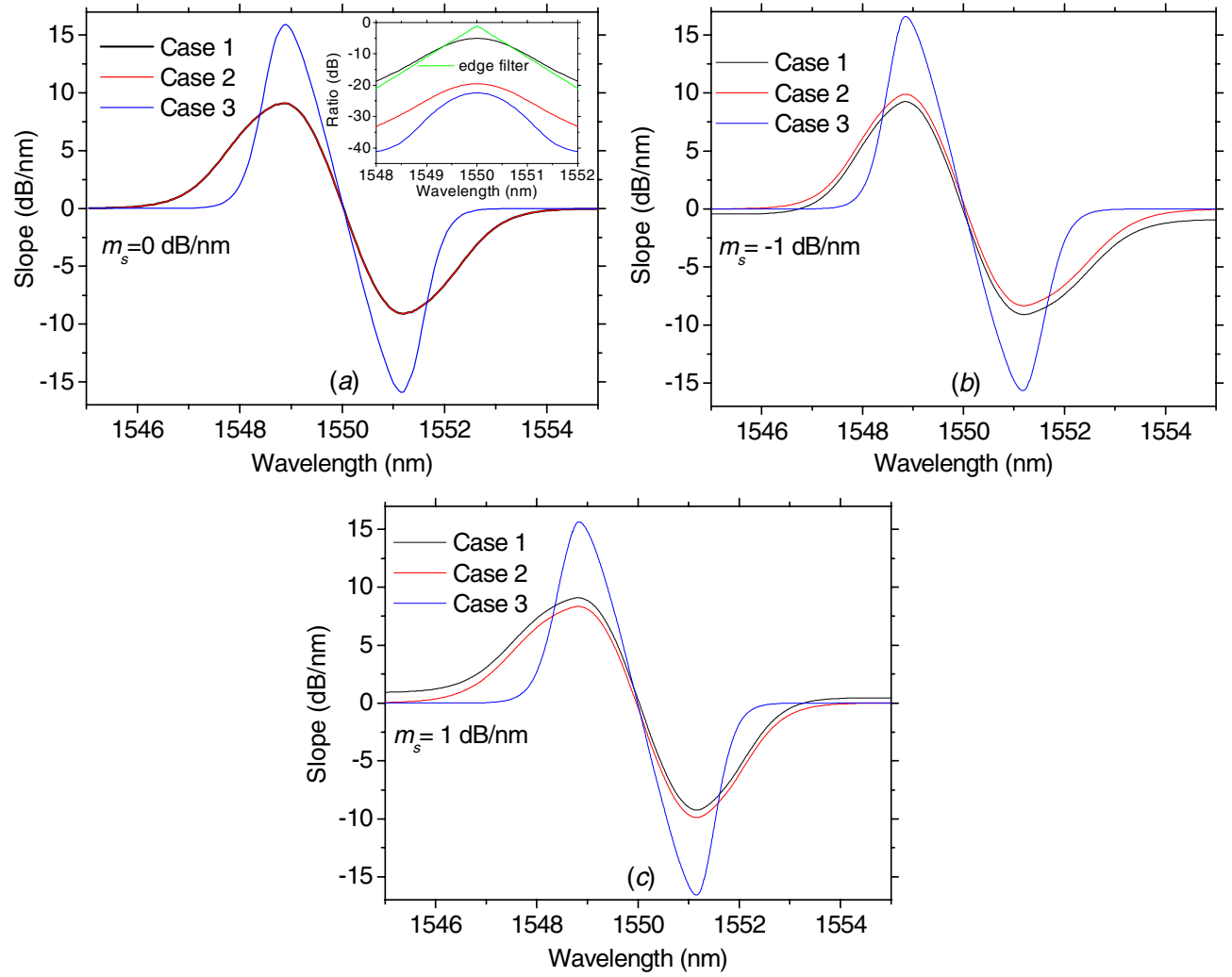

Figure 2. Simulated ratio slope in three cases with the optical source: (a) $m_{s}=0$, (b) $m_{s}=-1$ and (c) $m_{s}=1 \mathrm{~dB} \mathrm{~nm}^{-1}$.

are larger than the slope of the edge filter $\left(10 \mathrm{~dB} \mathrm{~nm}^{-1}\right)$ used in our simulations. This is because the signal passes twice through the edge filter, and hence the equivalent slope of the edge filter is twice that of the real one. This result indicates that with the same components in a ratiometric system, different physical arrangements will have different performances and that case 3 is likely to be the best arrangement of the three cases.

\subsection{Influence of the $S N R$ of $F B G$}

Figure 3 gives the simulation results of the influence of the SNR of FBG on the system ratio. In this simulation, we assume the output of the optical source is a constant. The other parameters are the same as above.

Figure 3 shows that the SNR of the FBG has a significant influence on the system performance. When the SNR of the FBG is $40 \mathrm{~dB}$, the maximum slope in cases 3 and 1 is 20 and $10 \mathrm{~dB} \mathrm{~nm}^{-1}$, respectively; when the SNR of the FBG drops to $20 \mathrm{~dB}$, the maximum slope in cases 3 and 1 also drops to 15.9 and $9.1 \mathrm{~dB} \mathrm{~nm}^{-1}$, respectively. Figure 3 also shows that the influence of the SNR of the FBG in case 3 is more significant than that in cases 1 and 2. When the SNR of the FBG is $40 \mathrm{~dB}$, in a wavelength range $5.18 \mathrm{~nm}$ (from 1547.4 to $1552.58 \mathrm{~nm}$ ) the slope in case 3 is larger than that in cases 1 and 2. However, when the SNR of the FBG drops to $20 \mathrm{~dB}$, the wavelength range where the slope in case 3 is larger than that in cases 1 and 2 drops to $3.26 \mathrm{~nm}$ (from 1548.36 to $1551.62 \mathrm{~nm})$.

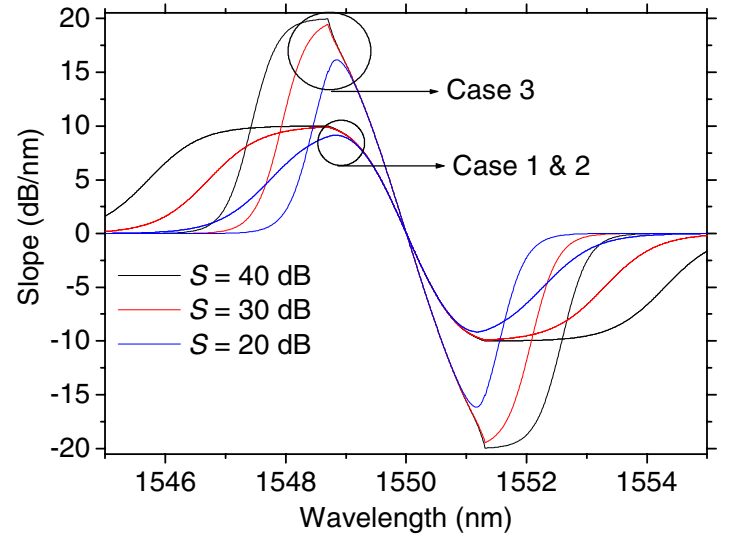

Figure 3. Simulated ratio slope in three cases with different SNR of the FBG.

\subsection{Influence of the slope of the edge filter}

The slope of the edge filter employed in the ratiometric system is an important factor which will influence the performance of the system. Figure 4 shows the simulation results for the influence of the slope of the edge filter on the ratiometric system. In the simulation, we assume the SNR of the FBG is $20 \mathrm{~dB}$ and the other parameters are the same as before. Figure 4 shows that as the slope of the edge filter increases from 5 to $10 \mathrm{~dB} \mathrm{~nm}^{-1}$, the maximum slope of the system in all three cases increases. It can be seen from figure 4 that the slope response versus wavelength in case 3 corresponding $m=$ $5 \mathrm{~dB} \mathrm{~nm}^{-1}$ overlapped with that in cases 1 and 2 corresponding 


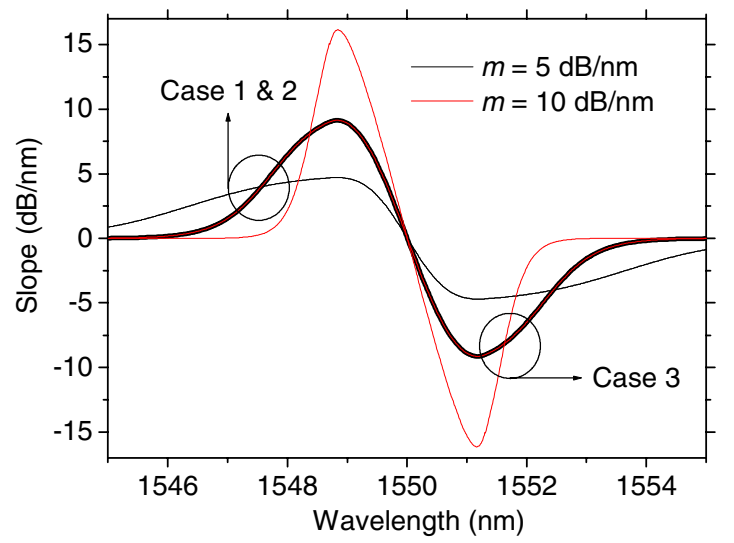

Figure 4. Simulated ratio slope in three cases with different slopes of the edge filter.

$m=10 \mathrm{~dB} \mathrm{~nm}^{-1}$, which indicates that the ratio response in case 3 employing an edge filter with slope of $m=5 \mathrm{~dB} \mathrm{~nm}^{-1}$ is the same as that in case 1 and 2 employing an edge filter with slope of $m=10 \mathrm{~dB} \mathrm{~nm}^{-1}$.

\section{Experimental verification}

Experimental verifications were carried out by using a FBG temperature sensor and a thin-film filter as an edge filter. The spectra of the thin-film edge filter, FBG and the measured ratio versus temperature are shown in figure 5.

In case 1 , the optical power in the reference arm is provided by the reflected FBG signal whose power is much lower than the broadband source which provides the reference power in cases 2 and 3 . The absolute value of the measured ratio is thus very different for case 1 compared to cases 2 and 3 . However, for comparison purposes for the measured ratios, the three curves are normalized to a common ratio value of $0 \mathrm{~dB}$ at $10{ }^{\circ} \mathrm{C}$. Figure 5 shows that as the temperature increases from 10 to $70{ }^{\circ} \mathrm{C}$, the measured ratio increases monotonically in all three cases. However, the rate of increase (corresponding to the slope) is significantly different. The discrimination in case 3 is the largest of the three cases indicating that case 3 has the best resolution. Figure 5 also shows that the maximum difference in the measured ratios between case 3 and other cases is circa $1.5 \mathrm{~dB}$, which agrees well with the simulated results in the inset to figure $2(a)$, given that (1)

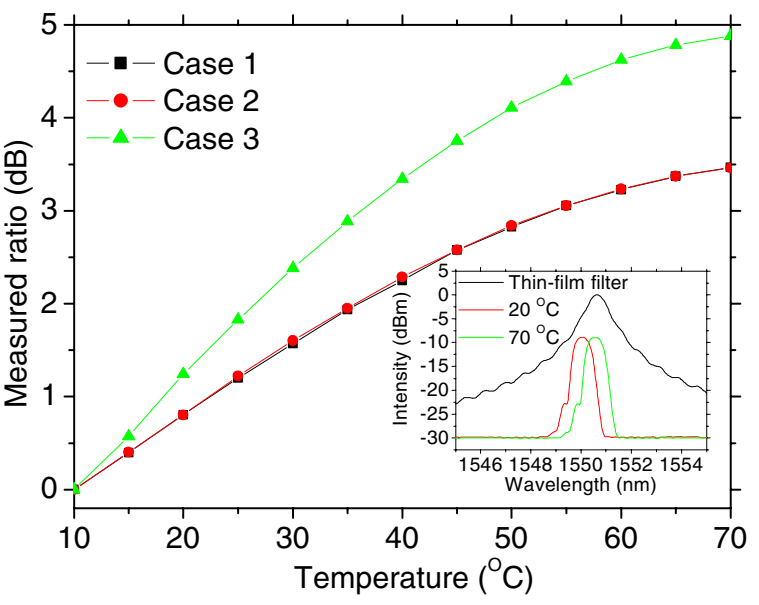

Figure 5. Measured ratio versus temperature and spectra of the thin film and FBG.

the maximum temperature-induced wavelength shifts in our experiments were relatively small (around $0.6 \mathrm{~nm}$, assuming a $10 \mathrm{pm}{ }^{\circ} \mathrm{C}^{-1}$ wavelength shift for the FBG) and (2) the FBG operates close to the peak of the triangular edge filter response.

To further investigate the effect of the different slopes (and hence the different resolutions) of the three cases, an incremental step change of $0.5{ }^{\circ} \mathrm{C}$ for a low-temperature range from $9{ }^{\circ} \mathrm{C}$ to $10^{\circ} \mathrm{C}$ and a high-temperature range from $69^{\circ} \mathrm{C}$ to $70{ }^{\circ} \mathrm{C}$ was applied to the FBG sensor over a period of $20 \mathrm{~s}$. The measured ratio variations versus time are shown in figure 6.

To allow for a valid comparison of the three cases, we have used separate but aligned graphs. Figure 6 shows that for both temperature ranges cases 1 and 2 have a similar resolution and case 3 has the best performance, which verifies our numerical simulations above.

\section{Conclusion and discussion}

The investigations on the effect of the position of the edge filter in a ratiometric wavelength measurement system were provided based on three cases: (1) the reflected FBG signal passes through both the reference arm and the edge filter arm, (2) the reflected FBG signal is connected directly to the edge filter arm and does not pass through the reference arm, (3) the

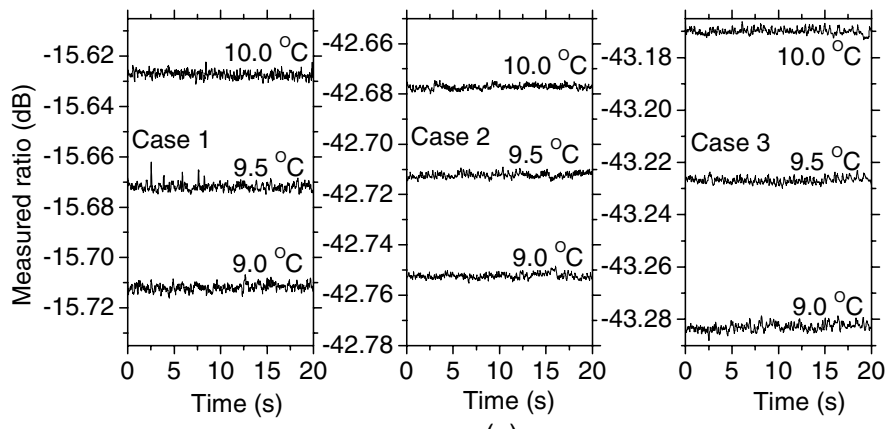

(a)
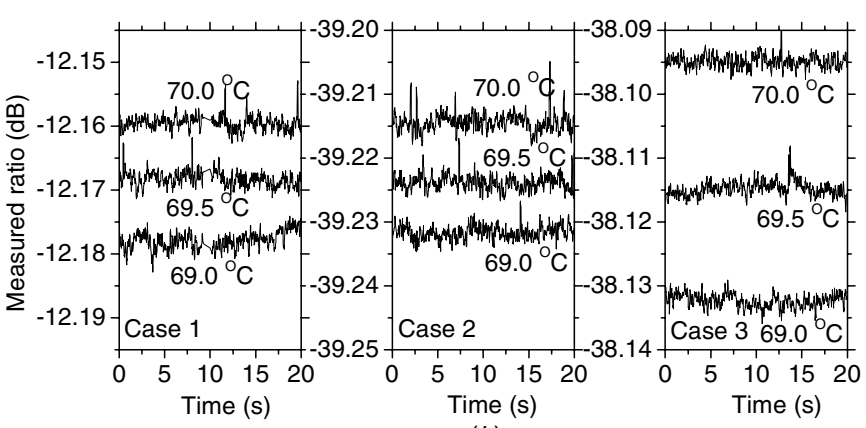

(b)

Figure 6. Measured ratio variations versus time at $(a) 9$ and $10{ }^{\circ} \mathrm{C}$ and $(b) 69$ and $70{ }^{\circ} \mathrm{C}$. 
edge filter sits in line with the FBG and thus the source power is filtered prior to reaching the FBG. Numerical simulations and experimental verifications were carried out and the conclusion is that case 3 could achieve the best performance and cases 1 and 2 have similar wavelength resolution but lower than that in case 3 . However, it should be noted that for realworld applications where component performance can drift with time, case 1 has the advantage that drift in circulator insertion loss does not affect system performance because of the referencing arrangement used. Cases 2 and 3 cannot compensate for circulator insertion loss drift and thus such drift may have a negative impact on system accuracy over time. If drift in the circulator insertion loss is found to be problematic, then case 1 can be used, but in order to achieve the same resolution as case 3 , an edge filter with a substantially higher slope will be required.

\section{Q3 References}

[1] Yu X, Shum P, Ren G B and Ngo N Q 2008 Photonic crystal fibers with high index infiltrations for refractive index sensing Opt. Commun. 281 4555-9

[2] Guo H R, Pang F F, Zeng X L, Chen N, Chen Z Y and Wang T Y 2008 Temperature sensor using an optical fiber coupler with a thin film Appl. Opt. 47 3530-4

[3] Kersey A D, Davis M A, Patrick H J, LeBlanc M, Koo K P, Askins C G, Putnam M A and Friebele E J 1997 Fiber grating sensors J. Lightwave Technol. 15 1442-63
[4] Lin Y B, Pan C L, Kuo Y H, Chang K C and Chern J C 2005 Online monitoring of highway bridge construction using fiber Bragg grating sensors Smart Mater. Struct. 14 1075-82

[5] Hongo A, Kojima S and Komatsuzaki S 2005 Applications of fiber Bragg grating sensors and high-speed interrogation techniques Struct. Control Health Monit. 12 269-82

[6] Melle S M, Liu K and Measures R M 1992 A passive wavelength demodulation system for guided-wave Bragg grating sensors IEEE Photonics Technol. Lett. 4 516-8

[7] Wu Q, Hatta A M, Semenova Y and Farrell G 2009 Use of a SMS fiber filter for interrogating FBG strain sensors with dynamic temperature compensation Appl. Opt. 48 5451-8

[8] Wang Q, Farrell G and Freir T 2005 Study of transmission response of edge filters employed in wavelength measurements Appl. Opt. 44 7789-92

[9] Kersey A D, Berkoff T A and Morsey W W 1993 Multiplexed fiber Bragg grating strain-sensor system with a fiber Fabry-Perot wavelength filter Opt. Lett. 18 1370-2

[10] Kersey A D and Berkoff T A 1992 Dual wavelength fiber interferometer with wavelength selection via fiber grating elements IEE Electron. Lett. 28 1215-6

[11] Wang Q, Rajan G, Farrell G, Wang P F, Semenova Y and Freir T 2007 Macrobending fibre loss filter, ratiometric wavelength measurement and application Meas. Sci. Technol. 18 3082-8

[12] Xu M G, Geiger H and Dakin J P 1996 Modeling and performance analysis of a fiber Bragg grating interrogation system using an acousto-optic tunable filter J. Lightwave Technol. 14 391-6 


\section{QUERIES}

\section{Page 1}

Q1

Author: Please be aware that the colour figures in this article will only appear in colour in the Web version. If you require colour in the printed journal and have not previously arranged it, please contact the Production Editor now.

\section{Page 2}

Q2

Author: The sense of the sentence 'Since the output of an optical source at...' is unclear. Please check.

\section{Page 5}

Q3

Author: Please check the details for any journal references that do not have a blue link as they may contain some incorrect information. Pale purple links are used for references to arXiv e-prints. 